\title{
Novel selective relaxant binding agent for the reversal of pipecuronium and rocuronium induced neuromuscular block
}

\author{
Fábián Á.I. ${ }^{1}$, Tassonyi E. ${ }^{1}$, Csernoch V. ${ }^{1}$, Fedor M. ${ }^{1}$, Szente L. ${ }^{2}$, Fülesdi B. ${ }^{1}$ \\ 1 University of Debrecen Clinical Center, Dept of Anaesthesiology \& Intensive Care, Debrecen, Hungary, \\ ${ }^{2}$ Cyclolab SA, Research and Development Department, Budapest, Hungary
}

\section{Background}

Despite advances in neuromuscular monitoring and increasing awareness, a significant portion of patients have postoperative residual neuromuscular block (PORNB) at the time of extubation and arrival in the PACU1.

PORNB significantly increases the risk of postoperative respiratory complications (hypoxia, aspiration, pneumonia) ${ }^{2,3}$ and is associated with a delayed PACU discharge 4 .

Reversal with cholinesterase inhibitors (ChEl) can cause severe side-effects ${ }^{5}$, including airway complications and efficacy is also a concern.

Sugammadex, a selective relaxant binding agent (SRBA), is effective for reversal of neuromuscular block caused by steroidal NMBAs ${ }^{6,7}$.

Reversal with sugammadex has been shown to be superior and more reliable ${ }^{8,9}$, than reversal with ChEls.

High cost limits the widespread use of sugammadex for routine end of surgery reversal of neuromuscular block.

Our workgroup therefore strives to develop more cost effective SRBAs.

The candidate molecule: Carboxy-Methyl-Gamma-Cyclodextrin (CMGCD).

Specifically designed to have a high affinity for pipecuronium.

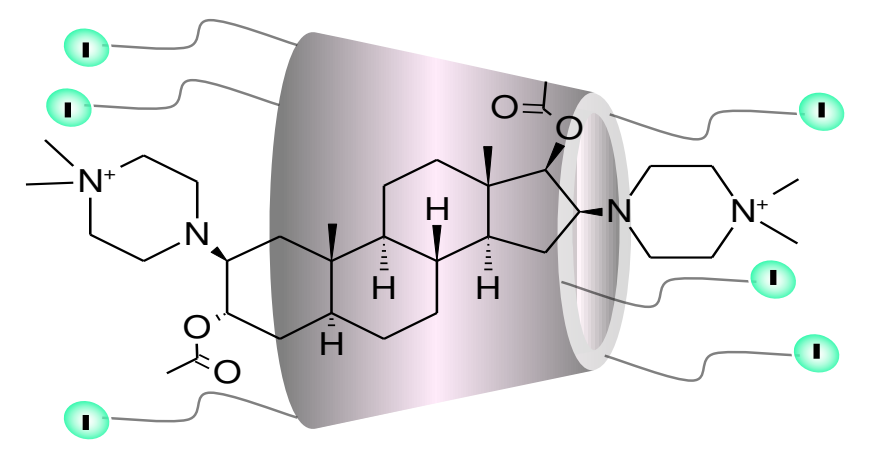

\section{Methods}

Male wistar rats were sacrificed, the hemi-diaphragm and associated phrenic nerve were excised.

The muscle-nerve preparation was suspended in an ISO-07-TSZ2D tissue holder (Experimetria Ltd., Hungary) in Krebs-buffer and aerated by bubbling $95 \% \mathrm{O}_{2}+5 \% \mathrm{CO}_{2}$ (Vol\%) through the solution. The tissue holder allowed independent electrical stimulation of the nerve and muscle. An isometric force-displacement transducer was attached to measure the contraction force of the diaphragm muscle after muscle relaxant and SRBA administration.

Cumulative dose-response curves were determined to calculate halfeffective concentrations $\left(\mathrm{EC}_{50}\right)$.

To better visualize SRBA affinity for NMBAs, SRBA EC Sn $_{50}$ values were normalized to the concentration of NMBAs, yielding affinity index (AI) values.

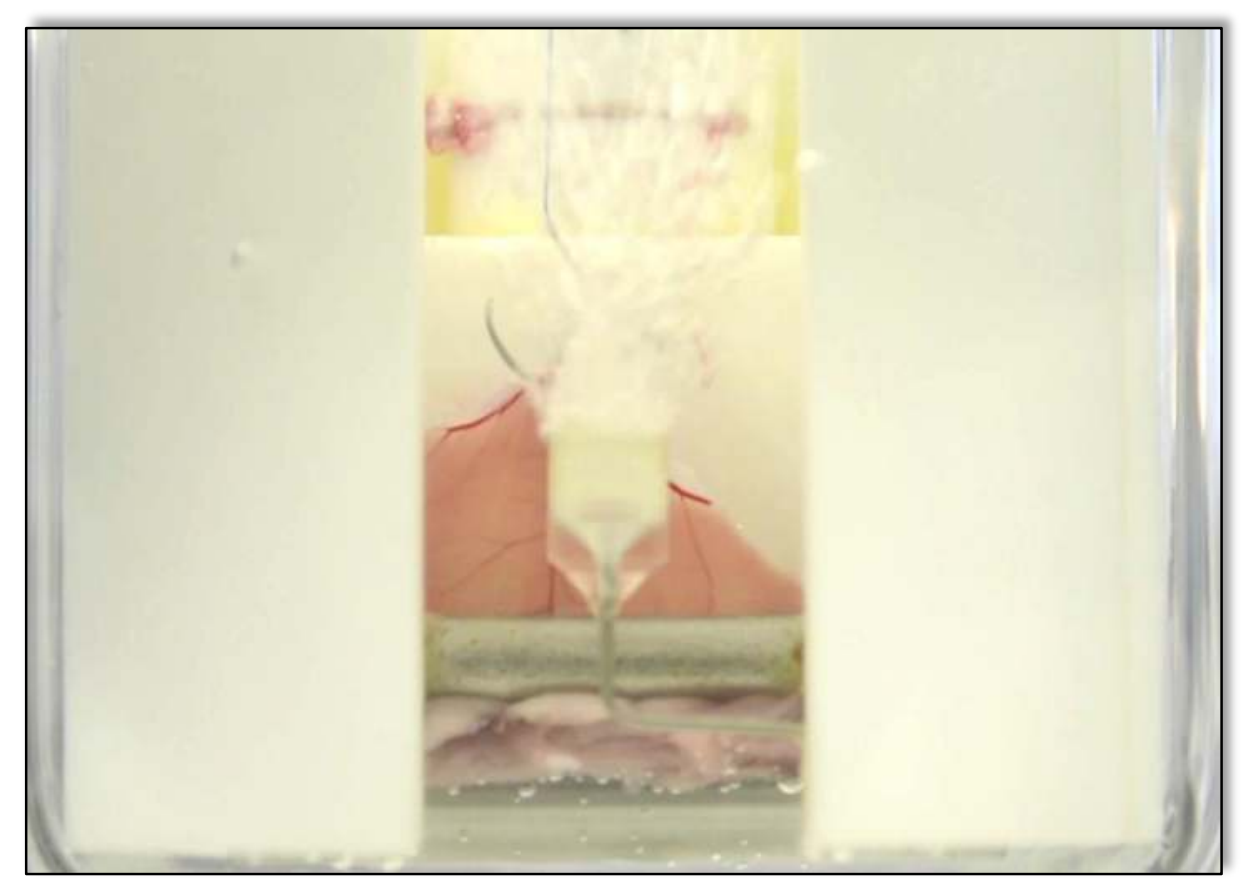

\section{Results}

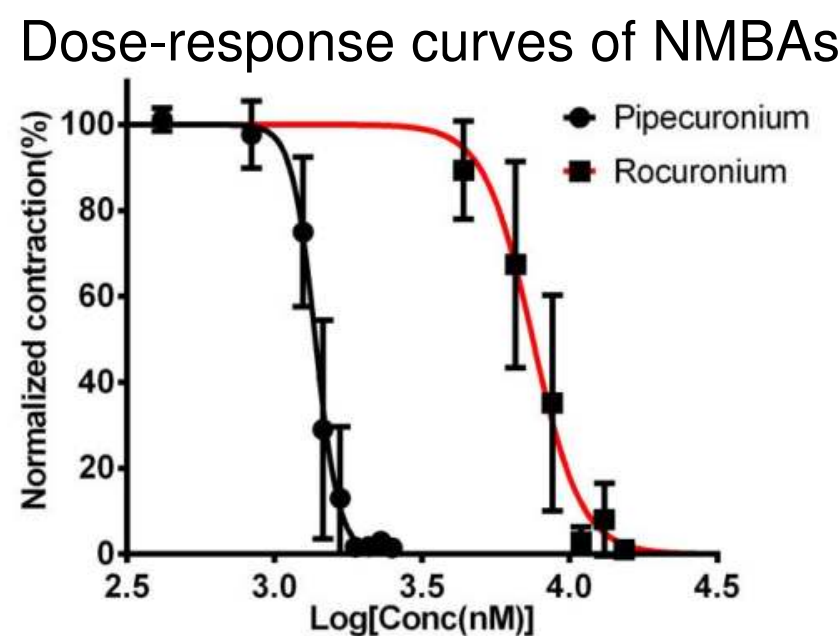

Reversal of rocuronium block

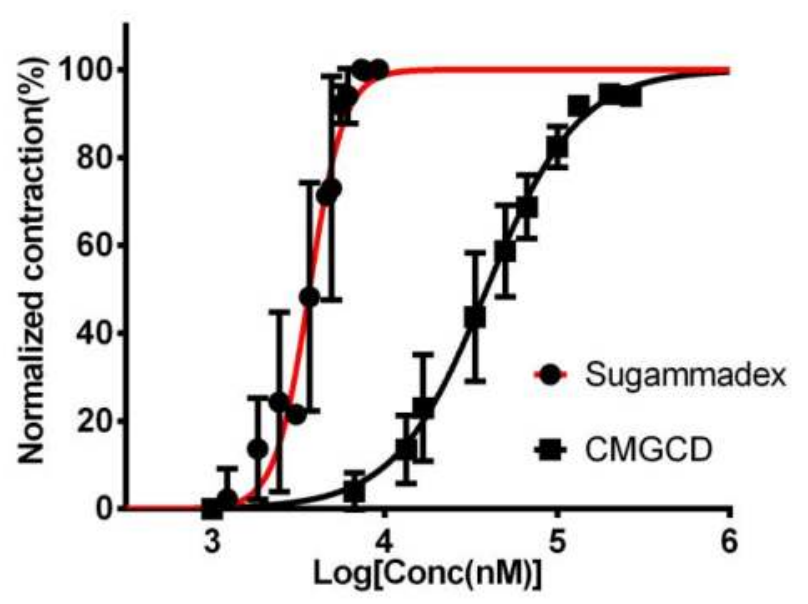

$\mathrm{EC}_{50}(\mu \mathrm{M})$ values for NMBAs

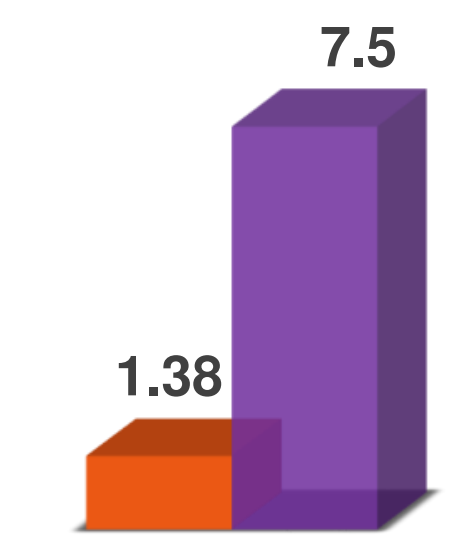

- Pipecuronium

- Rocuronium
Reversal of pipecuronium block

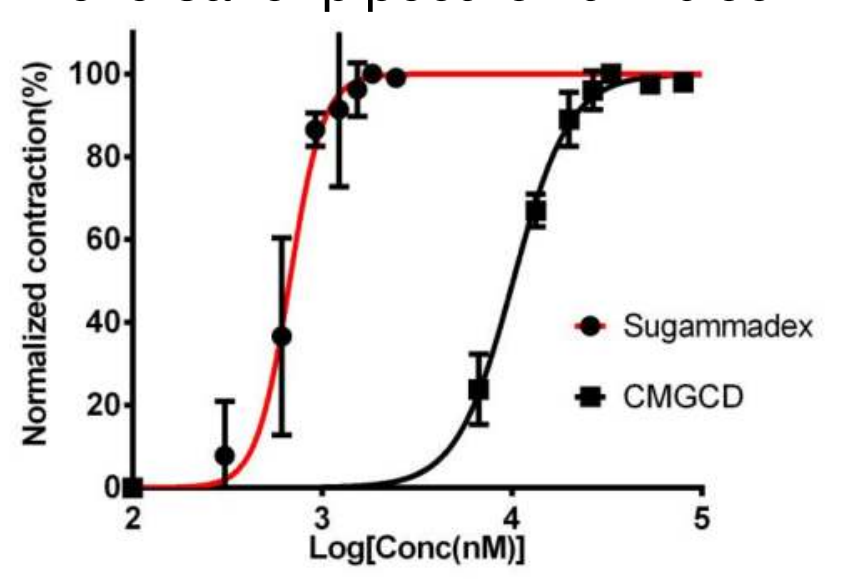

Time-course of block reversal

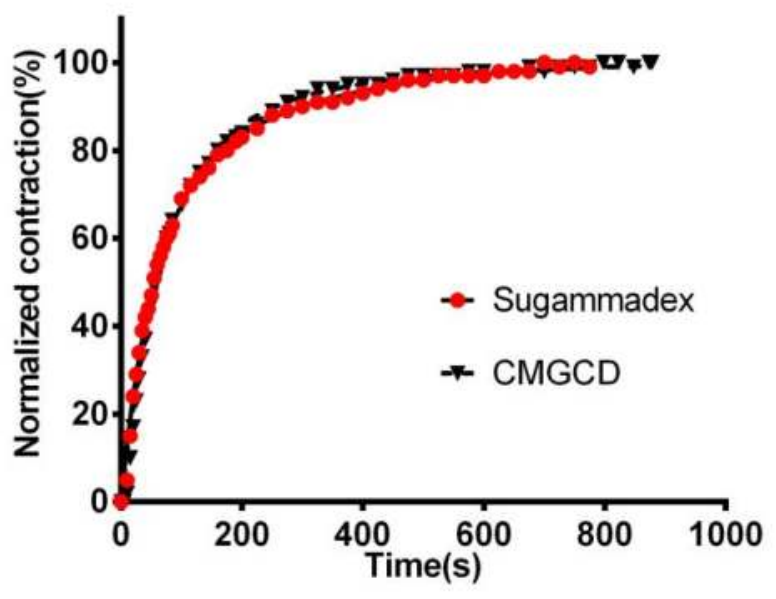

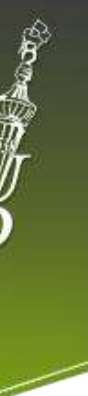

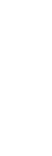

